Journal of Applied Fluid Mechanics, Vol. 9, Special Issue 1, pp. 139-145, 2016.

Selected papers from the $7^{\text {th }}$ International Exergy, Energy

and Environment Symposium, IEEE7-2015

Available online at www.jafmonline.net, ISSN 1735-3572, EISSN 1735-3645.

DOI: $10.36884 /$ jafm.9.SI1.25874

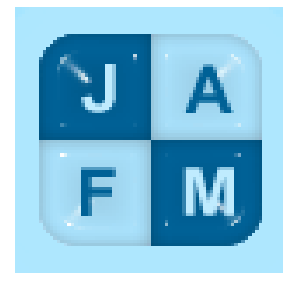

\title{
Forced Convection in a Cylinder Filled with Porous Medium, including Viscous Dissipation Effects
}

\author{
B. Zallama ${ }^{\dagger 1}$, L. Zili Ghedira ${ }^{1}$ and S.B. Nasrallah ${ }^{1}$ \\ ${ }^{1}$ Université de Monastir, École Nationale d'Ingénieurs de Monastir, Laboratoire LESTE, \\ Avenue Ibn El Jazzar 5019, Monastir, Tunisie
}

†Corresponding Author Email: zallama.boutheina@live.fr

(Received October 20, 2015; accepted December 10, 2015)

\begin{abstract}
In this work, a numerical study of forced convection of an incompressible fluid through a cylinder filled with a porous medium is carried out by taking into account the heat due to viscous dissipation. Dimensionless equations of the problem are solved numerically. The energy transport bidimensional model is based on the local non-thermal equilibrium assumption with consideration of viscous dissipation effects. The influence of various parameters like Darcy number, Reynolds number, Forchhheimer coefficient and Eckert number on temperature fields is investigated and examined throughout this paper. It is found that all these parameters have significant influence on thermal performance of the packed bed within certain conditions.
\end{abstract}

Keywords: Forced convection, Heat transfer, Cylindrical packed bed, Porous medium, Viscous dissipation.

\section{NOMENCLATURE}

\footnotetext{
$A^{-1}$ form factor

$B i \quad$ Biot number

$C p \quad$ specific heat at constant pressure

$D \quad$ cylinder diameter

Da Darcy number

Ec Eckert number

$F \quad$ Forchheimer coefficient, $F=\frac{1.75}{\sqrt{150 \varepsilon^{3}}}$

$H$ heat transfer coefficient ${ }^{1}$

$H_{f s} \quad$ interfacial heat transfer coefficient permeability of the porous medium

$K$ $K=\frac{\varepsilon^{3} d_{p}^{2}}{150(1-\varepsilon)^{2}}$

$L \quad$ cylinder height

$P^{*} \quad$ dimensionless fluid pressure

Pr Prandtl number

$r \quad$ radial component

Re Reynolds number

$\mathrm{R}_{\mathrm{k}} \quad$ thermal conductivity ratio

$\mathrm{S} \quad$ specific interfacial area

$\mathrm{T}$ temperature

U velocity
}

\section{INTRODUCTION}

The problem of heat transfer convection through porous media is frequently encountered in many

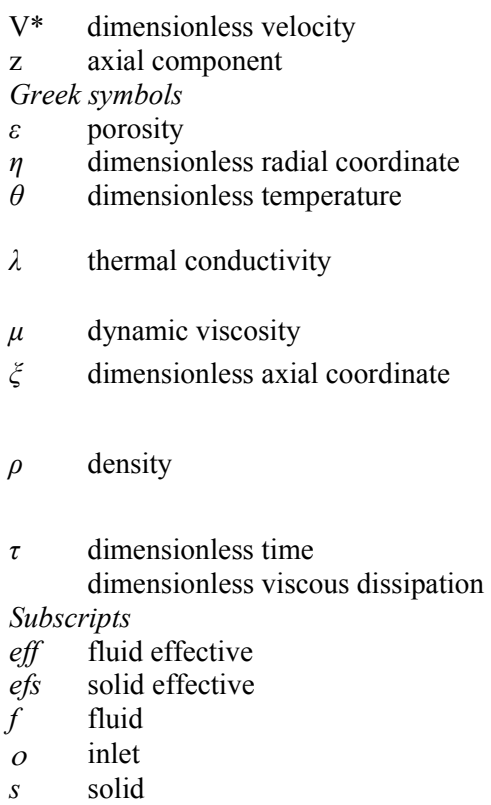

practical fields of science and engineering, such as thermal insulation in buildings, packed bed reactors, sensible heat-storage beds, chemical and petroleum engineering, thermal management of electronic 
cooling, improvement of performance of heat transfer systems and others. Over the last several decades, heat transfer in conducts fully and partially filled with porous media has been investigated experimentally, theoretically and numerically by many researchers. A comprehensive review of the subject was given by Nield et al. (1999). Ranganathann et al. (1984) and Chen et al. (1996) have studied the combined free and forced convection for vertical plates embedded in porous media. Kuznetzov et al. (2006) have used a perturbation approach to obtain analytical expressions of the velocity, temperature, and transient Nusselt number for the problem of forced convection with laminar pulsating flow in a saturated porous channel or tube. The porosity variation and the viscous dissipation effects were neglected in this study. Yee et al. (2002) have treated the viscous dissipation effects on forced convection heat transfer in cylindrical packed beds taking into account the porosity variation. Local thermal equilibrium was adopted and special emphasis was given to the heat generation due to viscous dissipation in forced convection liquid flow. It was found that, in the case of the adiabatic boundary, the effect of internal heat generation associated with viscous dissipation increases with the radius of the packed column to the particle diameter ratio and Reynolds number. An analytical study on fully developed forced convection in a homogeneous porous medium is developed by Hung et al. (2009). The temperature distributions in the transverse direction with the inclusion of frictional heating due to viscous dissipation are obtained, with variations of Darcy number and Brinkman number. The frictional heating effect on the temperature distributions is investigated and analyzed for both heating and cooling processes. Variations of Nusselt number as a function of Darcy number and Brinkman number are examined, and the deviations of the Nusselt numbers with that of the model without incorporating the effect of frictional heating are presented in a contour deviation map for a more holistic comparison.

Chen et al. (2011) have studied the effects of viscous dissipation on the temperature profiles for a fully developed forced convection flow between two parallel plates with a constant heat flux boundary condition. A two-equation model that includes viscous dissipation in the fluid phase is solved analytically and exact solutions for the temperature fields are obtained. Based on the solutions, the effects of several parameters on the transverse temperature profiles and Nusselt number are studied. The same authors (2011b) extended this study by including thermally developing effects. They found that the local heat transfer coefficients depend strongly on the relative thermal resistance to fluid and solid conduction and to a lesser extent on Brinkman number and porous medium shape factor.

Furthermore, there are controversial issues as reported in the literature by (Tso and Mahulikar 1998; Kim et al. 2000; Lee and Vafai 1999; Marafie and Vafai 2001; Al-Hadhrami et al. 2003; Nield 2000b, 2002; Amiri and Vafai 1994) on using which model to a better modelling and concretizing of heat transfer behavior in packed beds of porous media. In fact, there is not yet agreement between researchers on the appropriate form of the dissipation function. The viscous dissipation effect on forced convection in a porous saturated circular tube with an isoflux wall is investigated on the basis of the Brinkman flow model by Hooman et al. (2006). The case of a semi-infinite flat plate embedded in a saturated porous medium where the fluid is induced to move upwards by natural convection and during which viscous dissipation is considered is investigated by Amgad et al. (2013). Three terms were considered to contribute to viscous dissipation, namely Darcy's term, Forchheimer's term and Al-Hadhrami's term. The governing dimensional set of equations take into account the influence of the Darcy, Gebhart and Rayleigh numbers and the Forchheimer term on the temperature and velocity distributions. It is found that the irreversible process of transforming the kinetic energy of the moving fluid to viscous dissipation is very much influenced by the relative magnitude of these dimensionless parameters. Nield et al. (2004) performed the modelling of viscous dissipation in a porous medium saturated by an incompressible fluid and discussed the cases of Darcy, Forchheimer and Brinkman models. It has been demonstrated for the Forchheimer model that the viscous dissipation is represented by a term that is apparently independent of the viscosity, and this paradox is resolved. Finally, scale analysis is employed to estimate the importance of viscous dissipation in various circumstances. Transient hydrodynamic and heat transfer behavior of Newtonian fluid flow in vertical parallel-plate channels partially filled with a porous medium has been investigated numerically by Mastaneh et al. (2012). The influences of macroscopic local inertial term and the viscous heating due to the viscous dissipation were taken into account in the momentum equations of the porous region and the thermal energy equations, respectively. Moreover, Forchheimer-Brinkman extended Darcy model was used to model fluid flow in the porous region. The effects of the porous medium macroscopic inertial term and the viscous dissipation were investigated carefully. The predicted results clearly indicate that neglecting the inertial effect in high permeability porous media or high velocity flows can alter substantially the flow and heat transfer characteristics. An analytical study on fully developed forced convection in a homogeneous porous medium is reported by Hung et al. (2008). Incorporating the internal heating effect of viscous dissipation, closed form solutions of the temperature distributions in the transverse direction are obtained and analyzed for both heating and cooling processes. Variations of Nusselt number as a function of Darcy number and Brinkman number and the existence of singularity in Nusselt number are also discussed. Many refinements have been made to the equations used to model single-phase fluid flow and heat transfer in a saturated porous medium, such as inertial effects, boundary friction and viscous dissipation. Also, additional effects such as those due to rotation or a magnetic field are 
performed by Nield (2000).

The aim of this paper is to study the effect of viscous dissipation on forced convection heat transfer inside a cylinder filled with a saturated porous medium based on the local non-thermal equilibrium assumption. In fact, in this study, the Forchheimer extended Darcy model is considered. Therefore, the viscous dissipation effects are included in the energy equation. In this context, efforts are focused on identifying the effects of different dimensionless numbers on heat transfer, namely the Darcy number $\mathrm{Da}$, the Reynolds number $\mathrm{Re}$, the inertial parameter $\mathrm{F}$, and the Eckert number Ec. We investigate under which conditions the viscous dissipation terms should be considered and when their effects have a significant impact on heat transfers in porous media.

\section{Statement of The Problem}

In this study, heat transfer characteristics accounting for viscous dissipation within a cylinder filled with a porous medium as shown in (Fig. 1) is carried out. It is assumed that the circulating incompressible fluid is at forced convection flow and initially at uniform temperature and velocity. Furthermore, the fluid is considered Newtonian with constant physical properties. The porous medium is considered to be homogeneous, isotropic and in non-local thermal equilibrium with the saturated fluid. The cylinder wall is adiabatic. The effects of the different terms namely Darcy's term and Forchheimer's term, contributing to viscous dissipations in saturated porous media are performed and investigated. The influence of the particular dimensionless numbers namely Reynolds number, Darcy number, Forchhheimer coefficient and Eckert number is studied in details.

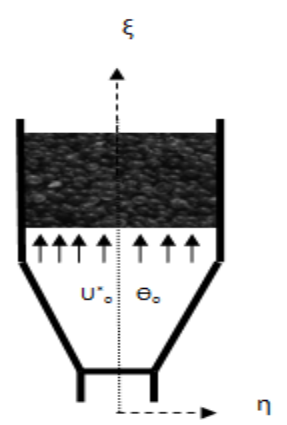

Fig.1. Schematic diagram of the problem and the coordinates system.

\section{ANAlysis}

Considering (Fig. 1), the equations of motion and the thermal energy equations for an unsteady-state forced convection flow through a cylinder filled with a porous medium can be expressed as:

\subsection{Governing Equations}

The governing equations are non-dimensionalized to provide the equations with groupings of numbers permitting the determination of the dominating effects in the handled phenomenon. Consequently, using the dimensionless parameters, the continuity and momentum dimensionless equations are expressed by:

$\frac{\partial V_{\xi}^{*}}{\partial \xi}+\frac{1}{\eta} \frac{\partial}{\partial \eta}\left(\eta V_{\eta}^{*}\right)=0$

$\frac{1}{\xi}\left(V_{\xi}^{*} \frac{\partial V_{\xi}^{*}}{\partial \xi}+V_{\eta}^{*} \frac{\partial V_{\xi}^{*}}{\partial \eta}\right)=-\frac{d P^{*}}{d \xi}$

$+\frac{1}{\operatorname{Re}} \frac{1}{\eta} \frac{\partial}{\partial \eta}\left(\eta \frac{\partial V_{\xi}^{*}}{\partial \eta}\right)-\frac{\varepsilon}{D a \operatorname{Re}} V_{\xi}^{*}-\frac{F \varepsilon}{\sqrt{D a}} V_{\xi}^{* 2}$

The dimensionless unsteady-state thermal energy equations for the fluid phase and the solid matrix are expressed respectively by:

$$
\begin{aligned}
& \frac{\partial \theta_{f}}{\partial \tau}+\frac{1}{\varepsilon} \frac{\partial}{\partial \xi}\left(V_{\xi}^{*} \theta_{f}\right)=\frac{B i}{\operatorname{Re} \operatorname{Pr} R_{k}}\left(\theta_{s}-\theta_{f}\right) \\
& +\frac{1}{\operatorname{Re} \operatorname{Pr}}\left[\frac{\partial^{2} \theta_{f}}{\partial \xi^{2}}+\frac{1}{\eta} \frac{\partial}{\partial \eta}\left(\eta \frac{\partial \theta_{f}}{\partial \eta}\right)\right]+\phi \\
& \frac{\partial \theta_{s}}{\partial \tau}=\frac{B i \alpha^{+}}{\operatorname{Re} \operatorname{Pr}}\left(\theta_{f}-\theta_{s}\right) \\
& +\frac{\alpha^{+}}{\operatorname{Re} \operatorname{Pr}}\left[\frac{\partial^{2} \theta_{s}}{\partial \xi^{2}}+\frac{1}{\eta} \frac{\partial}{\partial \eta}\left(\eta \frac{\partial \theta_{s}}{\partial \eta}\right)\right]
\end{aligned}
$$

Where is the contribution due to viscous dissipation that works as a distributed internal heat generation source. It is currently expressed as:

$\phi=\frac{E c}{D a \operatorname{Re}} V_{\xi}^{* 2}+\frac{F E c}{\sqrt{D a}} V_{\xi}^{* 3}$

Where,

$$
\begin{aligned}
& \operatorname{Re}=\frac{U_{o} D \rho_{f}}{\mu_{f}} \\
& D a=\frac{K}{D^{2}} \\
& \operatorname{Pr}=\frac{\mu_{f} C p_{f}}{\lambda_{f}} \\
& E c=\frac{U_{o}^{2}}{C p_{f} T_{o}} \\
& B i=\frac{h_{f s} s D^{2}}{\lambda_{s}}
\end{aligned}
$$

Thus, as can be seen immediately for uniform forced convection flows, which is the case here, the energy balance equations (3) and (4) will be associated to equation (5) to deal with the effect of viscous dissipation. From these equations, we notice that the dimensionless temperature distribution is dependent on pertinent non- 
dimensional parameters which will be analyzed in the following sections.

Moreover, theses equations are subject to the following dimensionless initial and boundary conditions:

Initially, we have:

$\theta_{f}(\tau=0, \xi, \eta)=0$

$\theta_{s}(\tau=0, \xi, \eta)=0$

The boundary conditions in dimensionless forms are:

$$
\begin{aligned}
& \theta_{f}(\tau, 0, \eta)=\theta_{o} \\
& \frac{-\partial \theta_{f}}{\partial \xi}(\tau, A, \eta)=\frac{H_{f} D}{\lambda_{e f f}} \theta_{f} \\
& \frac{\partial \theta_{f}}{\partial \eta}(\tau, \xi, 0)=\frac{\partial \theta_{s}}{\partial \eta}(\tau, \xi, 0)=0 \\
& \frac{\partial \theta_{f}}{\partial \eta}(\tau, \xi, 1)=\frac{\partial \theta_{s}}{\partial \eta}(\tau, \xi, 1)=0 \\
& \frac{-\partial \theta_{s}}{\partial \xi}(\tau, A, \eta)=\frac{H_{s} D}{\lambda_{e f s}} \theta_{s} \\
& \frac{\partial \theta_{s}}{\partial \xi}(\tau, 0, \eta)=\frac{H_{o} D}{\lambda_{e f s}}\left(\theta_{s}-\theta_{o}\right)
\end{aligned}
$$

Where $\mathrm{A}^{-1}=\mathrm{D} / \mathrm{L}$ is the form factor; $\mathrm{L}$ is the cylinder height.

$\mathrm{H}_{\mathrm{o}}$ and $\mathrm{H}_{\mathrm{s}}$ are the solid exchange coefficients in the entrance and at the exit of the cylinder, respectively. $\mathrm{H}_{\mathrm{f}}$ is the fluid exchange coefficient at the exit of the cylinder.

\section{Solution Procedure}

Computational technique for the resolution of the considered equations along with the imposed boundary conditions is performed by using finite differences method for flow equations and finite volumes method for heat transfer equations based on a uniform grid structure in the computational space. The latter consists in defining a network of points on the considered medium and volumes of control around each point. The equations are integrated on these volumes. The fully implicit scheme is used for the time derivatives. Line by line iterative method is used to solve the obtained algebraic equations system. The obtained discretized equations are numerically solved and then results are discussed.

\section{RESUlTS AND DisCUSSION}

The resolution of the differential set of equations depends on the dimensionless variables namely, the Reynolds number Re, the Prandtl number Pr, the Biot number $\mathrm{Bi}$, the Darcy number $\mathrm{Da}$ and the Eckert number Ec. The followings are the ranges considered in this study for the different dimensionless groups: $500 \leq \mathrm{Re}<1 \mathrm{E}+5$, $1 \mathrm{E}-6 \leq$ $\mathrm{Da} \leq 1 \mathrm{E}-9,1 \mathrm{E}-9 \leq \mathrm{Ec} \leq 1 \mathrm{E}-5,0 \leq \mathrm{F} \leq 4.5,0.4 \leq \mathrm{Pr} \leq$ 2 and $2 \mathrm{E}+3 \leq \mathrm{Bi} \leq 4 \mathrm{E}+4$. The effects of each one of these dimensionless parameters are investigated by fixing all other parameters values.

\subsection{Effects of Ec/ Re number}

As the viscous dissipation due to Darcy's term is expressed within a specific number: $\mathrm{Ec} / \mathrm{Re}$, the influence of this term for the temperature distribution is investigated and analyzed. In fact, Reynolds number describes the relative intensity of the inertial forces compared with viscous forces. Thus, higher values of Re number imply higher intensity of inertial forces and vice versa, while the Eckert number is useful in determining the relative importance in a heat transfer situation of the kinetic energy of a flow. It is the ratio of the kinetic energy to the enthalpy driving force for heat transfer. We consider $2 \mathrm{E}-12 \leq$ $\mathrm{Ec} / \mathrm{Re} \leq 2 \mathrm{E}-10$. The temperature distributions for the various entertained values are plotted in (Fig. 2). The other parameters were set as follows: $\mathrm{Da}=1 \mathrm{E}-9, \mathrm{~F}$ $=4.5, \mathrm{Bi}=20000, \operatorname{Pr}=1.2$ and $\mathrm{K}=0.01$.

Figure 2 shows the effects of $\mathrm{Ec} / \mathrm{Re}$ ratio on the profiles of the non-dimensional temperature. It is observed that the temperature increases with time with a maximum attained at $\tau=1200$ and finally remains constant. It is also observed that the temperature increases with increasing values of the defined ratio. We can even notice that, for $\mathrm{Ec} / \mathrm{Re}<$ 5.E-11, there is no contribution of the viscous dissipation terms on the heat transfer. Such contribution is, indeed, really significant for $\mathrm{Ec} / \mathrm{Re}$ $\geq 1 \mathrm{E}-10$. Therefore, as Ec/Re increases, the generated viscous dissipation increases. This may be explained by the fact that this term is multiplied by the fluid friction term in the energy equation leading to large kinetic energy available to be converted to heat through the viscous dissipation.

Moreover, the temperature distribution across the packed bed is illustrated in (Fig. 3). The effects of viscous dissipation terms for $\mathrm{Ec} / \mathrm{Re}=2 \mathrm{E}-10, \mathrm{Da}=$ $1 \mathrm{E}-9, \mathrm{~F}=4.5, \mathrm{Pr}=1.2, \mathrm{~K}=0.01$ and $\mathrm{Bi}=20000$ are studied. It is obvious that the frictional heating is much important as the flow passes through the porous media. Thus, the heat transfer due to frictional heating temperature is increased with the increase of the porous media's height, leading to more pronounced heat generation as $\xi$ further increases.

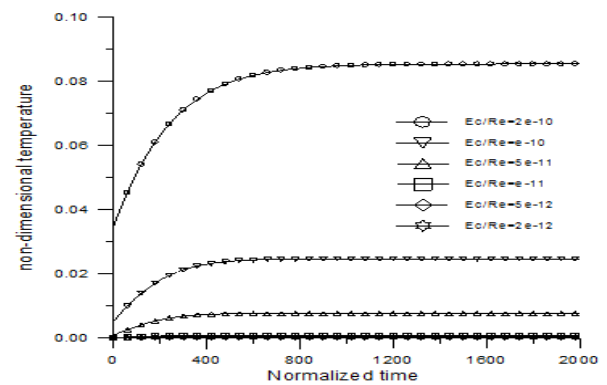

Fig. 2. Dimensionless fluid temperature profiles at the bed exit for $D a=1 E-9, F=4.5$ at different Ec/Re. 


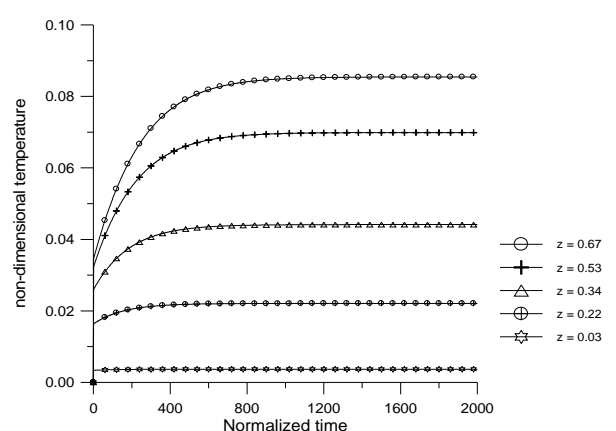

Fig. 3. Dimensionless fluid temperature profiles across the bed for $\mathrm{Ec} / \mathrm{Re}=2 . \mathrm{E}-10, \mathrm{Da}=1 \mathrm{E}-9$, $\mathrm{F}=4.5$.

\subsection{Effects of Darcy's number}

In this section, the effect of Darcy number on viscous dissipation is investigated. We consider 1E$9 \leq D a \leq 1 E-6$. Whithin this range, viscous dissipation has shown to be significantly affected by the change of Da. (Fig. 4) shows the outlet temperature distribution for various values of $\mathrm{Da}$ with the other parameters set at $\mathrm{Ec} / \mathrm{Re}=2 \mathrm{E}-10, \mathrm{~F}=$ $4.5, \mathrm{Pr}=1.2, \mathrm{~K}=0.01$ and $\mathrm{Bi}=20000$. It is clear from this figure that the temperature increases with time. It depicts also that the Darcy number decrease enhances the effect of the fluid friction resulting in higher generated viscous dissipation. It is absolutely clear that the viscous dissipation generation is getting dramatically pronounced for small Darcy number, implying that the internal heating effect intensifies with decreasing Da. Indeed, as Darcy decreases, so as the permeability decreases, the viscous dissipation increases due to high restrictive medium. Therefore, it can be concluded that the contribution of Darcy becomes much more considerable for $\mathrm{Da} \leq 1 \mathrm{E}-8$, i.e., lower values of Darcy number lead to smaller convection in a more restrictive medium. Therefore, the internal heat generation increases and consequently, the fluid temperature increase becomes higher. On the opposite side, it follows that larger values of Darcy reveals that the permeability is large (approaching clear fluid scenario), and then, the internal heat generation is much less intense, even insignificant.

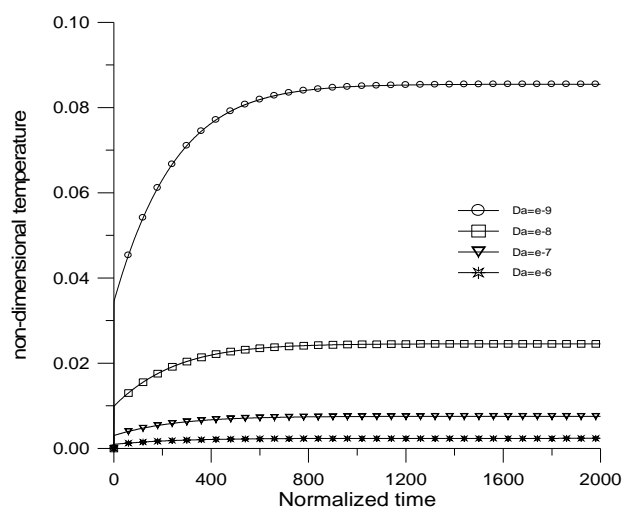

Fig. 4. Dimensionless fluid temperature profiles at bed exit for $E c / R e=2 E-10, F=4.5$ at different

Da.

\subsection{Effects of the non-Darcy parameter}

The non-Darcy parameter F defines, generally, the structural properties of the saturated porous medium continuum. It equals the ratio of pressure drop caused by gas-solid interactions to that by viscous dissipation. In this study, temperature distributions for various values of $\mathrm{F}$ at $\mathrm{Ec} / \mathrm{Re}=2 \mathrm{E}-10, \mathrm{Da}=1 \mathrm{E}$ $9, \operatorname{Pr}=1.2, \mathrm{~K}=0.01$ and $\mathrm{Bi}=20000$ are plotted in (Fig. 5). The different values undertaken, here, are $0,0.5,1.5,2.5$ and 4.5. From this figure, it is clear that the temperature distribution keep the same profiles shown previously, and, manifest a progressive increase in the generated heat with the increase of F. Higher values of F imply higher drag forces. Otherwise, the inertial effect (with increasing F) intensifies resistance to flow (Lee and Vafai, 1999) which further increases the internal heat generation and temperature.

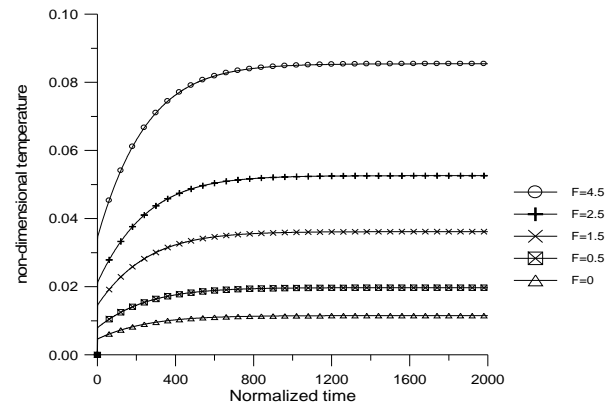

Fig. 5. Dimensionless fluid temperature profiles at bed exit for $\mathrm{Ec} / \mathrm{Re}=\mathbf{2 E - 1 0 ,} \mathrm{Da}=1 \mathrm{E}-9$ at different $F$.

\subsection{Effects of the Porosity}

The influence of the solid matrix porosity, as a basic characteristic of a porous medium, on the convection heat transfer is presented in (Fig. 6). The study is performed for three different values of porosity, namely $0.1,0.2$ and 0.3 . It is obvious that the distribution of the outlet dimensionless temperature is considerably influenced by the bed porosity. A comparison of the three undertaken bed porosities effects on the outlet non-dimensional temperature reveals an increase of $10.8 \%, 2.4 \%$ and $0.9 \%$ respectively, of fluid outlet temperature with regard to the inlet one. Therefore, it is clear that the viscous dissipation is more significant as the porosity is lower.

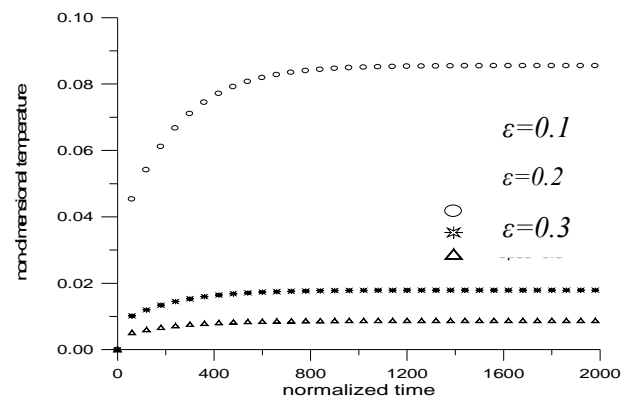

Fig. 6. The effects of the porosity on heat transfer distribution for $E c / R e=2 E-10, F=4.5$, Da=1E-9. 


\section{Conclusions}

This study has successfully determined the temperature profiles for forced convective heat transfer in a cylinder filled with a porous medium when taking into account the effects of viscous dissipation and local non-thermal equilibrium between solid and fluid phases. The effect of various parameters like Darcy number, Reynolds number, Biot number, Eckert number and Forchheimer parameter were investigated. The main findings are:

- Higher $\mathrm{Ec} / \mathrm{Re}$ values lead to high inertial forces, resulting in heat generation enhancement by frictional heat generation.

- As Darcy decreases, the permeability decreases and the viscous dissipation increases due to high restrictive medium.

- The temperature increases with an increase in inertial term, which acts as a flow resistance in the porous region. Otherwise, higher values of $F$ imply higher drag forces and consequently higher heat generation due to viscous dissipation.

Otherwise, it is obvious that the energy dissipation has a significant effect on the heat transfer characteristics that should be managed to enhance the performance of the processes.

Moreover, it is found that the heat transfer in porous medium is dramatically influenced by the variation of the bed porosity. Indeed, it is clear that the viscous dissipation is more significant as the porosity is low. Reducing the porosity increases the interior tortuosity of the porous medium, leading to an increase of the friction factor.

\section{REFERENCES}

Al-Hadhrami, A. K., L. Elliot and D.B. Ingham (2003). A new model for viscous dissipation in porous media across a range of permeability values. Transport Porous Med. 53, 117-122.

Al-Hadhrami, A. K., L. Elliott, and D.B. Ingham (2003). A new model for viscous dissipation across a range of permeability values. Transp. Porous Media. 53, 117-122.

Amiri, A. and K. Vafai (1994). Analysis of dispersion effects and non-thermal equilibrium, non-Darcian, variable porosity incompressible flow through porous media. Int . J. Heat Mass Transfer. 37, 939-954.

Chen, C. H., T. S. Chen and C. K. Chen (1996). Non-Darcy mixed convection along non isothermal vertical surfaces in porous medium. Int. J. Heat Mass Transfer 39, 1157-1164.

Chen, G. M. and C. P. To (2011). Forced convection with viscous dissipation using a two-equation model in a channel filled by a porous medium, International Journal of Heat and Mass Transfer, 54, 1791-1804.

Chen, G. M. and C. P. Tso (2011). A two-equation model for thermally developing forced convection in porous medium with viscous dissipation. International Journal of Heat and Mass Transfer. 54, 5406-5414.

Hajipour,M. A. M. Dehkordi (2012). Transient behavior of fluid and heat transfer in vertical channels partially filled with porous medium: Effects of inertial term and viscous dissipation. Energy Conversion and Management. 61, 1-7.

Hooman, K., A. Pourshaghaghy and A. Ejlali (2006). Effects of viscous dissipation on thermally developing forced convection in a porous saturated circular tube with an isoflux wall. Appl. Math. Mech (English ed.). 27, 617626.

Hung, Y. M., and C. P. Tso (2009). Effects of viscous dissipation on fully developed forced convection in porous media. International Communications in Heat and Mass Transfer. 36 (6), 597-603.

Hung,Y. M. and C. P. Tso (2008). Temperature variations of forced convection in porous media for heating and cooling processes: internal heating effect of viscous dissipation. Transp. Porous Med. 75, 319-322.

Kim, S. J., D. Kim and D.Y. Lee (2000). On the local thermal equilibrium in microchannel heat sinks, Int. J. Heat Mass Transfer. 43, 17351748.

Kuznetsov, A. V. and D. A. Nield (2006). Forced convection with laminar pulsating flow in a saturated porous channel or tube. Transp. Porous Media. 65, 505-523.

Lee, D. Y. and K. Vafai (1999). Analytical characterization and conceptual assessment of solid and fluid temperature differentials in porous media. Int. J. Heat Mass Transfer . 42, 423-435.

Lee, D. Y. and K. Vafai (1999). Analytical characterization and conceptual assessment of solid and fluid temperature differentials in porous media. International Journal of Heat and Mass Transfer. 42, 423-435.

Marafie, A. and K. Vafai (2001). Analysis of nonDarcian effects on temperature differentials in porous media. Int. J. Heat Mass Transfer. 44, 4401-4411.

Nield, D. A. (2000), Modelling Fluid Flow and Heat Transfer in a Saturated Porous Medium. Journal of Applied Mathematics \& Decision Sciences. 4(2), 165-173.

Nield, D. A. (2000). Resolution of a paradox involving viscous dissipation and nonlinear drag in a porous medium, Transp. Porous Media. 41, 349-357.

Nield, D. A. (2002). Modelling fluid flow in saturated porous media and at interfaces, In: 
B. Zallama et al. / JAFM, Vol. 9, Special Issue 1, pp. 139-145, 2016.

D.B. Ingham and I. Pop, eds. Transport Phenomena in Porous Media II. London: Pergamon, 1-19.

Nield, D. A. (2004). Comments on 'A new model for viscous dissipation in porous media across a range of permeability values. Transport in Porous Med. 55, 253-254.

Nield, D. A. and A. Bejan (1999). Convection in Porous Media. $2^{\text {nd }}$ edn. New York: SpringerVerlag.

Nield, D. A., A. V. Kuznetsov and M. Xiong, (2004). Effects of viscous dissipation and flow work on forced convection in a channel filled by a saturated porous medium. Transport Porous Med. 56, 351-367.

Ranganathann, P. and R. Viskanta (1984). Mixed convective boundary layer flow along a vertical surface in a porous medium. Num Heat Transfer, 7, 305-317.

Salama, A., I. A. Abbas, M. F. El-Amin and S. Sun (2013). Comparison study between the effects of different terms contributing to viscous dissipation in saturated porous media. International Journal of Thermal Sciences. 64, 195-203.

Tso, C. P. and S. P. Mahulikar (1998). The use of the Brinkman number for single phase forced convective heat transfer in microchannels. Int J. Heat Mass Transfer. 41, 1759-1769.

Yee, S. S. and K. Kamiuto (2002). Effects of viscous dissipation on forced- convection heat transfer in cylindrical packed-beds. Int. J. Heat Transfer. 45, 461-464. 